\title{
The design and implementation of high-speed data interface based on Ink-jet printing system
}

\author{
Yeli Li, Likun Lu*, Binbin Yan \\ Beijing Key Laboratory of Signal and Information Processing for High- \\ end Printing Equipments, \\ Beijing Institute of Graphic Communication, \\ Beijing 102600, China \\ lklu@bigc.edu.cn
}

\begin{abstract}
.
Ink-jet printing is an important part of the digital printing system; it has been widely used in various fields because of its wide adaptability, high-speed intelligent and low pollution. This paper chooses a kind of print head produced in XAAR company which works in dropping on demand manner. Firstly we introduce the hardware design of interface circuit and so on. Then we realize the functional modules of high-speed data interface of ink-jet printing system. At last, we focus on the design and implementation of high-speed data interface of ink-jet printing system.
\end{abstract}

Keywords: XAAR 1001, Ink-jet printing system, High-speed data interface, Hardware.

\section{Introduction}

Ink-jet printing is an important part of the digital printing system; it has been widely used in various fields because of its wide adaptability, high-speed intelligent and low pollution. As the diversification of printing ink and printing medium, ink-jet printing has expanded the connotation of the traditional printing. With the continuous development of ink-jet printing, many ink-jet devices appear on the market. Not only many foreign products enter China's market, many domestic enterprises are also developing its own ink-jet system. 
The data path of the ink-jet printing system has an important influence on printing quality, not only affect the image clarity, longitudinal/transverse coherence, but also plays an important role for printing speed. So the data transmission speed of the ink-jet printing affects the performance of the control system directly.

\section{XAAR 1001 print head}

The Xaar 1001 utilises Xaar's print head patented greyscale technology, enabling it to fire multiple drip sizes. An individual printhead prints at $360 * 360$ dpi.Xaar's grayscales technology provides the ability for the print head to produce droplets of ink with different ink volumes. By varying the number of sub-drops the actual drop volume on the substrate can be varied. The Xaar 1001 print head is built up from the following basic components as shown in Figure 1.

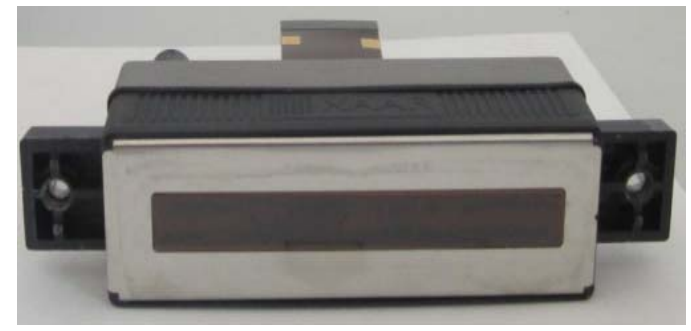

Fig. 1 Xaar 1001 print head

There are a total of 1020 nozzles visible on the nozzle plate. This comprises 2 rows of 510 nozzles each. Each row has 5 guard nozzles at either end, some of these guard nozzles are electronically addressable, but should not be used and hence there are a total of 1000 usable nozzles.

\section{The hardware block diagram of Ink-jet printing system}

According to the function, Ink-jet printing control system is divided into six parts: host computer, variable data transmission channel, embedded RIP processing card, control board, embedded processing card and data path between nozzles. As shown in Figure 2. 


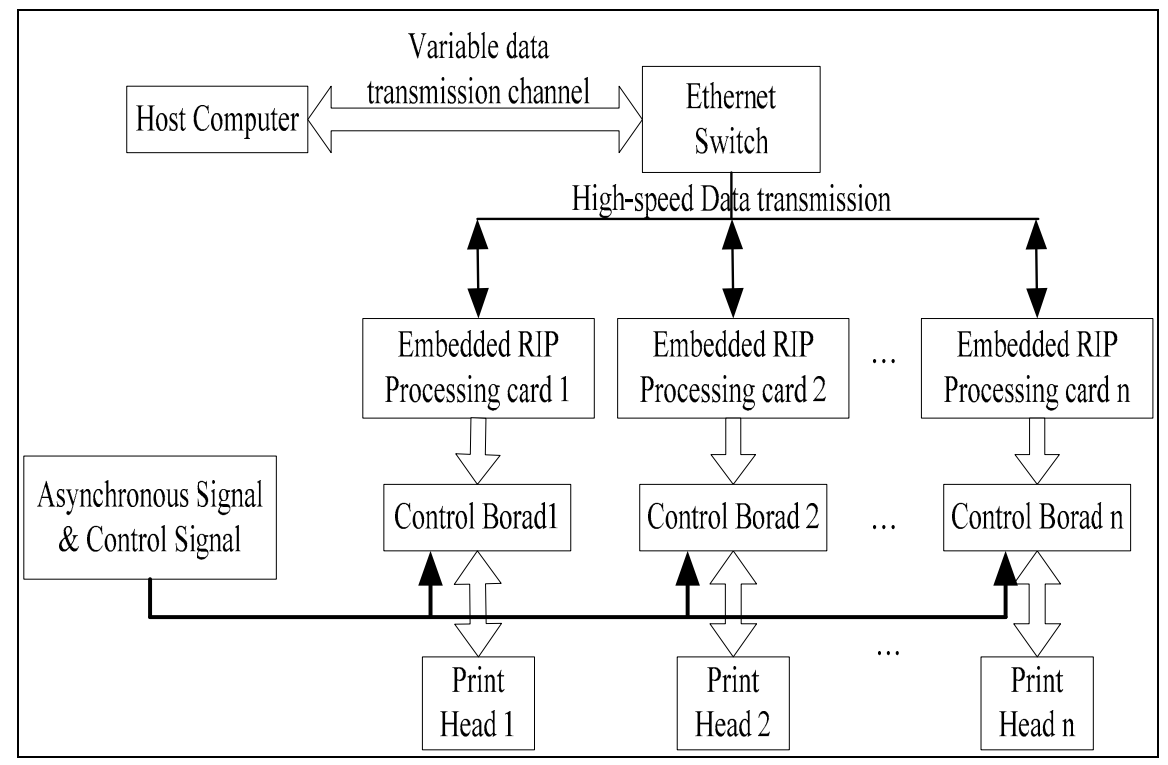

Fig. 2 The Block Diagram of Ink-jet Printing system

The architecture employs parallel embedded RIP processing card ${ }^{[1,2]}$. The specific format information (variable bar code or OCR character) is transmitted by high-speed variable data path to the embedded RIP processing card. After the control board receives the image data and control command, according to the control timing of the print head, send the print data to the ASIC within the print head. And to control the ignition frequency and drop size according to the parameters, then we can complete the image printing in the medium.

\section{The ASCI in the print head}

The print head utilises eight custom ICs (ASIC) per side in a two-bank serial arrangement. Each side is arranged in exactly the same way and hence the print head has a rotational symmetry through the axis shown in Figure3. The ASIC has two discrete areas for loading Configuration Data and Print Data and these areas are accessed by the DAT/nCFG signal being set to either high or low(DataHigh, Configuration-Low). The print head requires 8 independent ASIC supplies 
plus VCC per side, if chip trim support is required in the application, otherwise a single supply per side per ASIC is acceptable.

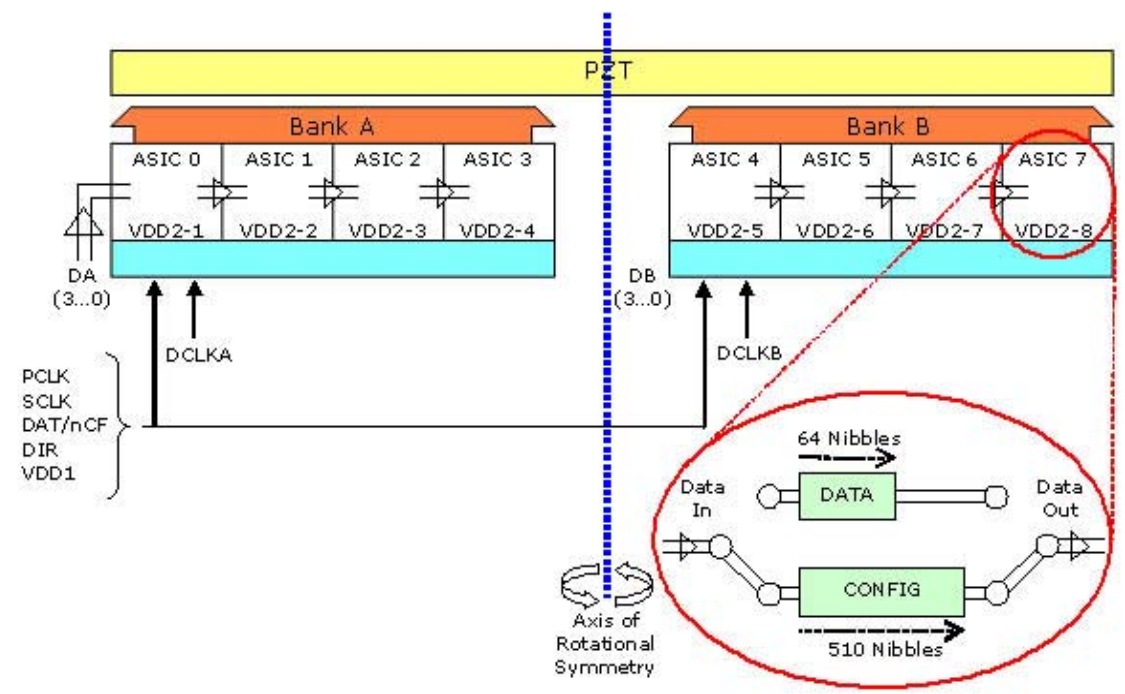

Fig. 3 ASIC Data Flow

The supply of signals in excess of the stated ratings may result in intermittent functionality or permanent failure and therefore must not be exceed.

\section{The transmission protocol}

The transmission protocol is shown in Figure 4. Print data and various control signals are transmitted to FPGA through the interface protocol and each bit is transferred via two differential signals by serial input mode. After the data packets received, according to the type of data packets, the data is divided into configuration data, image data, control signal, fire message, idle message and so on. These parameters are passed to the control board in the form of control message. Once the parameters are received by the control board, we need to transfer configuration data to print head. When the configuration data are received, the data should be stored in the buffer firstly, and then transferred to print head according to timing sequence. After the print head start to print, the 
image data is transferred to FPGA through interface protocol. The ignition signal is generated by the rotary encoder and photoelectric sensor, and then the print data is presented on the medium in the form of ink droplets.

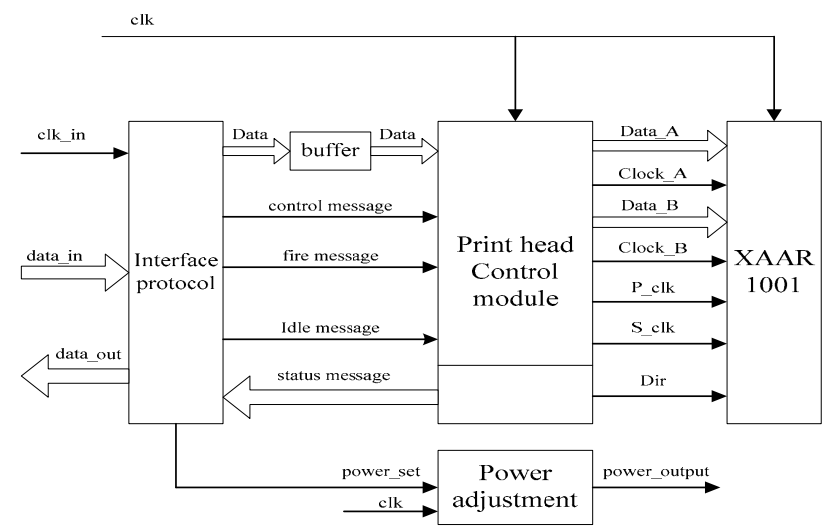

Fig. 4 The transmission protocol

Messages passed by the transmission protocol are encoded using a $4 \mathrm{~B} / 5 \mathrm{~B}$ scheme; this scheme enables message synchronization and allows Pixel, Control, and Data messages to be overlapped. Figure 5 shows data stream with overlapping messages using priority pre-emption.

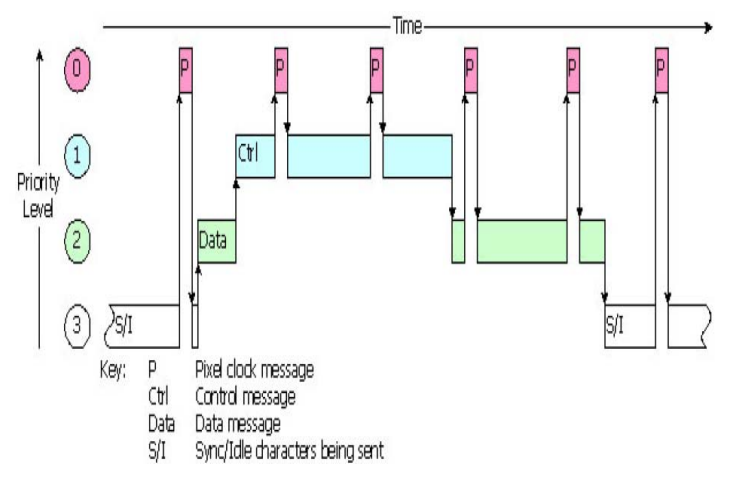

Fig. 5 The priority pre-emption of the data stream

The diagram starts and ends with the serial stream in its low-priority "Sync/Idle" mode. The green block labeled "Data" shows medium-priority Data messages pre-empting the "Sync/Idle" mode. The blue block labeled "Ctrl" shows a high-priority Control messages that has pre-empted the image data. The 
blocks shown in red labeled "P" are immediate-priority Pixel messages, these are punctuated throughout the data stream and pre-empt all other message types.

The interface protocol can safely transition from one message type to another and back again with no loss of data, using special characters defined within the 4B/5B scheme. Data and Control messages can be met under all conditions. Individual encoder clocks on a per side basis, allow sub pixel delays to the handled within the protocol.

During print head boot-up, a further message type is used. The use of a bootup character subset means that the Pixel, Control and Data messages are not supported during print head boot-up.

Sync/Idle characters should be transmitted whenever the serial bus is idle, i.e. not sending Data, Control or Pixel messages. It is also possible to send Sync/Idle characters periodically to help maintain data stream synchronization, for example, inserted into a long Data message.

The longest and most frequent message type are Data messages; as a result they have the lowest priority of any message that transfer actual data to the print head and thus can be pre-empted by both Control and Pixel messages. Any unsolicited data nibbles that are transmitted are considered by the print head to be part of any previous data message and will be loaded into the print head.

Control \& Status message contain nibbles that define an escape sequence, command ID, target address, length count and up to 16 additional payload nibbles. Status messages are only available within the uplink data stream and therefore, Status messages cannot be pre-empted and are always pulse width modulated.

Pixel messages are short( 2 characters) and subject to extremely low latency in order to ensure drop placement accuracy when print head are fired. Two pixel clock subtypes must be used to ensure that data is loaded correctly when operating in cycle trigger mode. The first pixel clock subtype indicates the first cycle in the 3-cycle sequence and triggers a data load when the pixel clock has been completed. The second pixel subtype indicates the subsequent second and third pixel clocks in the 3 -cycle sequence. 
Print head boot-up messages are used to configure the printheads FPGA before Pixel, Control and Data messages become active. Data stream should be managed by the drive electronics in accordance with Table 1 .

Table 1 XSPI messages and their priorities

\begin{tabular}{|l|l|l|l|l|}
\hline $\begin{array}{l}\text { Message } \\
\text { Type }\end{array}$ & Link & $\begin{array}{l}\text { Priority } \\
\text { Description }\end{array}$ & Priority & Interrupts \\
\hline Pixel & Down & Immediate & 0 & $3,2,1$ \\
\hline Control & Down & High & 1 & 3,2 \\
\hline Data & Down & Medium & 2 & 3 \\
\hline Sync & Down & Low & 3 & - \\
\hline Status & Up & - & - & -- \\
\hline Boot-up & Down & - & - & - \\
\hline
\end{tabular}

\section{Summary}

This paper analyzes the present situation and development trend of the ink-jet printing, and introduces the types of the ink-jet printing briefly. Firstly, we introduce the hardware design of the ink-jet printing system, including the selection of the print head, the design of interface circuit and so on. Then we apply the wavelet transform and 4B/5B encoder/decoder to design and realize the function modules of the ink-jet printing system. At last, we focus on the design and implementation of high-speed data interface of the ink-jet printing system.

\section{Acknowledgements}

This work was supported in part by the Science and Technology Project of Beijing Education Committee (SQKM201410015007), Program funded by the Education Commission of Beijing (TJSHG201310015016).

\section{References}


[1] B. Q. Zhang and S. X. Yang, J. The implementation of the circuit of the frequency-multiplier and phase discrimination in the FPGA. Industry and Mine Automation. 8, 69 (2005).

[2] X. J. Shi and H. Q. Li, J. The circuit of four frequency-multiplier and phase discrimination count based on CPLD in the application of montion controller. Manufacturing Technology and Machine Tools. 8, 85 (2008).

[3] Y. W. Liu, C. H. Li and T. S. Jin, J. The image denoising based on wavelet threshold value and sub-band with enhancement. Journal of Xiamen University, 2, 68 (2011).

[4] L. F. Qiao and Z. G. Wang, The design and verification of Verilog digital system, Publishing House of Electronics Industry, Beijing (2009).

[5] J. H. Wu and H. L. Cai, The design and Altera FPGA/CPLD, Post and Telecom Press, Beijing (2011).

[6] W. Y. Xia, The tutorial of the Verilog digital system design, Beijing University of Aeronautics and Astronautics Press, Beijing(2008).

[7] D. L. Donoho and L. M. Johnstone, J. Adapting to unknown smoothness via wavelet shrinkage. American Statistical association journal. 1200 (1995)

[8] S. Mallat and W. L. Hwang, J. Singularity detection and processing with wavelet. IEEE Trans on IT. 617 (1992). 